\title{
APUNTES PARA UNA TEORÍA DE LA FORMACIÓN
}

\author{
Javier Marrero Acosta* \\ Juan José Sosa Alonso** \\ Universidad de la Laguna
}

\section{RESUMEN}

En este trabajo, de reflexión teórica, partiendo de la constatación de la importancia creciente de las actividades que identificamos como «formación» en las sociedades posmodernas, se trata de justificar la idea de que la expresión española «formación» es un término confuso, polisémico y que, tras cada uno de los matices con que se puede emplear, se esconde todo un discurso de reflexión teórica, tremendamente abigarrado. Se trata de hacer un análisis y clarificación acerca de fuentes de confusión, identificadas en dos tipos fundamentales: de naturaleza terminológica y de naturaleza conceptual. Se concluye ofreciendo un marco interpretativo que permita ubicar e identificar las diferentes actividades de formación de manera integrada y se alerta acerca de la conveniencia de valorar las diferentes actividades que se presentan como formativas, desde una perspectiva crítica, puesto que no todo lo que se identifica como "formación» posee las mismas connotaciones y significados.

Palabras Clave: formación, formabilidad, Bildung, educación, formatividad.

\section{NOTES FOR A THEORY OF FORMATION}

\section{Abstract}

In this theoretical reflection work, we start from the confirmation of the growing importance of the activities that we identify as "formación" in postmodern societies. It is posted the idea that the Spanish expression "formación" is a confusing, polysemic term. and that, behind each of the nuances with which it can be used, hides a whole discourse of theoretical reflection, tremendously variegated. In this paper we try to maki an analysis and clarification about sources of confusion, identified in two fundamental types: terminological and conceptual in nature. The paper concludes by offering an interpretive framework that allows the different activities covered by the Spanish term of "formación" to be located and identified in an integrated manner and alerts about the advisability of evaluating the different activities that are presented as "formación", from a critical perspective, since not everything that is identified as "formacion" it has the same connotations and meanings. KEYwords: training, Bildung, bildsamkeit, education, formativity. 


\section{INTRODUCCIÓN}

La expresión «formación» se ha convertido, quizá, en una de las más repetidas y referenciadas en las sociedades del s. XXI. Este hecho no es sorprendente, ni inesperado. Ya en 1996 -hace 25 años- la Comisión Europea, con motivo del Año Europeo de la Educación y la Formación Permanentes, publicaba el libro blanco Enseñar y Aprender. Hacia la Sociedad del Conocimiento (Comisión Europea, 1996), en el que se afirmaba que «la educación y la formación serán, más que nunca, los principales vectores de identificación, pertenencia y promoción social. A través de la educación y la formación, adquiridas en el sistema educativo institucional, en la empresa, o de una forma más informal, los individuos serán dueños de su destino y garantizarán su desarrollo» (Comisión Europea, 1995, p. 16).

Sin duda, este mensaje ha calado en los países miembros, tanto en sus gobiernos como en sus políticas y en los diferentes sectores que conforman el tramado social, de manera que la importancia y omnipresencia de la formación (y de la educación) no ha dejado de crecer.

Esta percepción de presencia e importancia creciente de las cuestiones relacionadas con la formación puede ser verificada (de manera burda, si se quiere, pero eficaz), sin más que realizando una consulta en bases de datos contrastadas como la Web Of Science (WOS) o SciELO (Scientific Electronic Librari Online), a las que se ha tenido acceso a través de la Fundación Española para la Ciencia y la Tecnología (FECYT).

Restringiendo la búsqueda a publicaciones aparecidas desde 1980 hasta la actualidad, y sólo en los ámbitos o áreas más relacionados con la formación como objeto de estudio en sí mismo (se han excluido las publicaciones relativas a formación especializada, "formación en...», en el campo de la medicina, la ingeniería, las artes, la literatura, las ciencias en general, etc.), aparecen un total de 8755 publicaciones (La figura 1 detalla su distribución por áreas temáticas o campos de conocimiento).

Más interesante que el propio volumen de publicaciones registrado en los últimos 40 años es la evolución de las mismas, tanto en lo que se refiere a número de publicaciones por año como a número de citas por año (ver figura 2).

Muchas son las posibles explicaciones de este auge e importancia creciente de la formación (el propio libro blanco de la Comisión Europea ofrecía un amplio abanico de explicaciones). De entre todas ellas, queremos destacar la que consideramos más nuclear o subyacente a todas las restantes: la formación se relaciona con la idea de "saber» (en su sentido más amplio).

Lyotard (1991), al reflexionar sobre la evolución del «saber» en las sociedades del conocimiento, predecía que el concepto de saber, su significado, su sentido,

\footnotetext{
* Departamento de Didáctica e Investigación Educativa, Universidad de la La Laguna. E-mail: jmarrero@ull.edu.es.

** Departamento de Didáctica e Investigación Educativa, Universidad de la La Laguna. E-mail: jsosalo@ull.edu.es.
} 


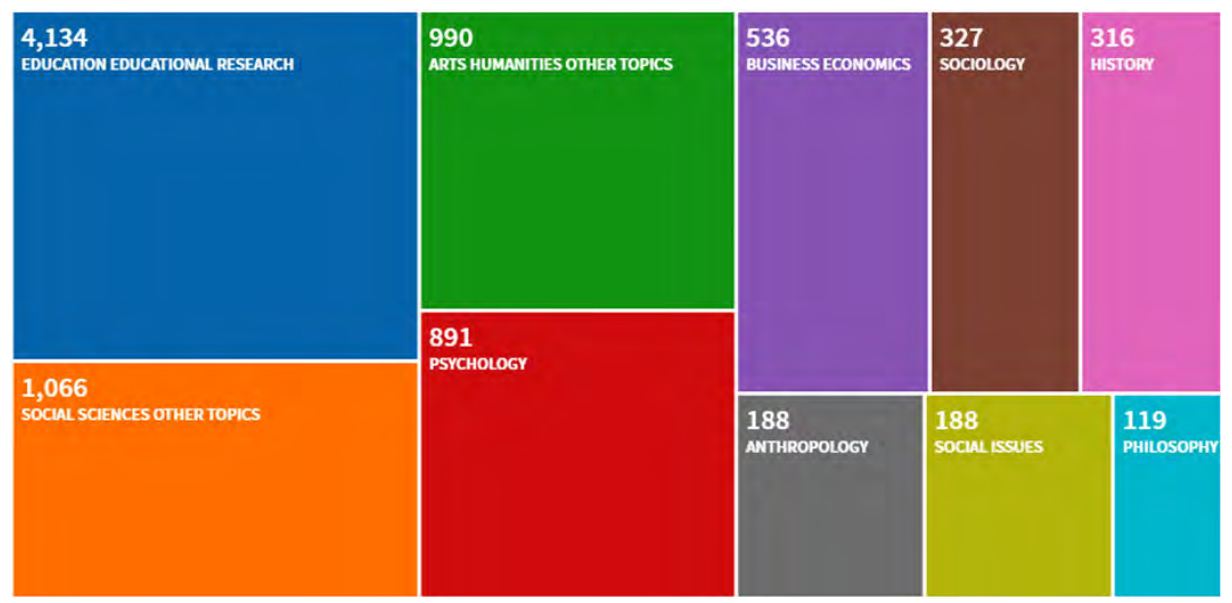

Fig. 1. Representación, por áreas temáticas o campos de conocimiento de las 8755 publicaciones obtenidas relacionadas con la palabra de búsqueda «formación» en WOS y ScieLO.
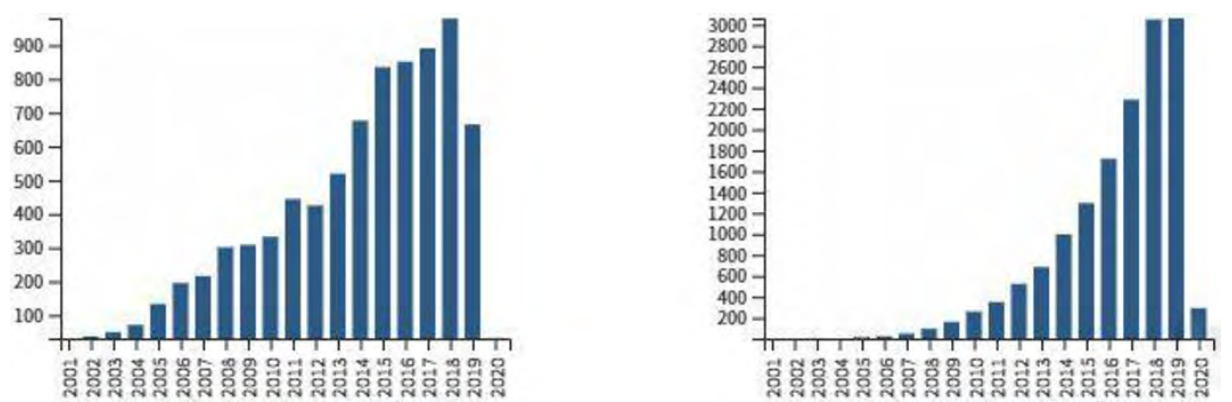

Fig. 2. Evolución de publicaciones y citas relativas a «formación» en los últimos 20 años (fuente: WOS y SciELO).

su valor... se iban a ver afectados de manera importante con el paso a la era posindustrial y posmoderna. Haciendo buena esa predicción, podemos afirmar que el saber es, hoy ya, un producto de consumo. Ha dejado de ser un fin en sí mismo y ha adquirido «valor de canje». En el concierto de las naciones, la economía del «saber» se ha convertido en un mecanismo indispensable para la potencia productiva. Las naciones ya pelean por dominar las informaciones y su sistema de producción (recuérdese el conflicto entre EE. UU. y China por el dominio de las tecnologías $5 \mathrm{G}$ y el veto americano a la empresa Huawey).

En este escenario, la formación, como tecnología de producción del saber, ha ganado un altísimo valor de demanda y se ha situado en el mismo centro de las políticas de desarrollo estratégico de todos los países. 
Pero ¿qué formación?... El propio Lyotard (1991) ya respondía, en parte, a esta cuestión al decir que «se puede esperar una potente exteriorización del saber con respecto al "sabiente" [...]. El antiguo principio de que la adquisición del saber es indisociable de la formación (Bildung) del espíritu, e incluso de la persona, cae y caerá todavía más en desuso».

En este caso, se podría decir que se ha evolucionado desde una concepción de la formación entendida como "tecnología del yo" (Foucault, 2008) a una idea de formación como tecnología de producción de saber, exteriorizado del concepto de persona, disociado de la idea original de formación como desarrollo humano y, por tanto, bastante desvirtuado de manera intrínseca.

La formación, buena parte de las actividades que englobamos bajo este concepto, en tanto que generan y proporcionan "conocimiento", han pasado a ser un «activo» clave en el desarrollo empresarial y en las dinámicas «de mercado». En palabras de Martín Alcázar (1999, p. 163): «Si la formación, en el campo educativo, nos remite al hombre individual y a su capacidad para decidir sobre su verdadero destino, en la empresa nos sumerge en el colectivo, nos disuelve en la organización. $\mathrm{Si}$ para la educación, la formación es la realización del valor, es ética, para el empresario es política con finalidad de producción».

En una línea similar apunta Mollenhauer $(1990 ; 143)$ cuando, tras recordar la afirmación de Horkheimer de que «el camino de la formación es un camino de enajenación (...); que no se forma uno por lo que hace de uno mismo, sino únicamente en la entrega a la causa tanto en el trabajo intelectual como en su praxis consciente», acaba preguntándose hasta qué punto se constata ese enajenamiento en la vida cotidiana del trabajo y cómo debe situarse al respecto el teórico de la formación.

Sin embargo, para poderlo hacer, para poder situarnos, como teóricos de las ciencias de la educación, frente al fenómeno de la formación, el primer problema con el que nos enfrentamos es la proliferación del uso de la expresión «formación», con interpretaciones bastante divergentes entre sí, dependiendo del contexto en que se use.

\section{«FORMACIÓN»: UN TÉRMINO CONCEPTUALMENTE CONFUSO}

Pareciera que, precisamente por su omnipresencia, la idea y el significado asociados a la expresión «formación» están ya más que superados. Que resulta innecesario y superfluo dedicar unas líneas a su clarificación. Nosotros, sin embargo, consideramos que este esfuerzo está más que justificado.

En el ámbito teórico, desde las ciencias de la educación, es ya clásica la referencia a Menze (1980) cuando señala, en relación con el concepto de formación, la posibilidad de identificar tres posturas, dos de las cuales abogan por su eliminación del vocabulario técnico pedagógico, por su potencial de confusión e imposibilidad de clarificación (más allá de posiciones metafísicas, ideológicas o subjetivas), y porque no es posible mantener la tesis de que la formación designe un proceso orgánico de desarrollo humano, del cual se desconocen las condiciones y procesos por los que ocurre. A estas dos posiciones, negacionistas de la formación, como constructo y concepto teórico en el ámbito de las ciencias de la educación, Menze agrega la existencia 
de «una inflación por lo que hace al empleo de "formación" y sus compuestos, que muestran cuán ambiguamente se habla hoy de formación» (Menze, 1980, p. 269).

Esta ambigüedad conceptual en torno a la formación, que ya vemos que no es nueva, no ha hecho más crecer a lo largo de este tiempo, con características añadidas propias, como veremos más adelante, en el contexto académico de habla hispana.

Un ejemplo de hasta dónde puede llegar esta confusión es el trabajo de Aguilar, Martínez, Fandiño y Fajardo (2007) que, tratando de hacer un relato prospectivo y retrospectivo de los procesos de formación en las organizaciones, hace un esfuerzo de clarificación del término «formación». En ese intento, los autores (siguiendo a Del Pozo, 1997) sitúan a la formación como un subtipo de los «procesos de educación» que ocurren en las organizaciones, junto con el entrenamiento, la capacitación, el desarrollo de personal, la información, la instrucción o el adoctrinamiento. En todo caso, lo identifican con una actividad muy relacionada con los procesos de aprendizaje y enseñanza y, partiendo de esta concepción, identifican y presentan ¡41! definiciones o concepciones de «formación», de otros tantos autores y autoras, recopilados entre 1990 y 2007.

Otro ejemplo lo tenemos en las siguientes dos citas extraídas de dos textos legales diferentes, ambos con rango de Ley Orgánica y ambos dedicados a regular aspectos relacionados con la formación y sistemas de formación en España:

LO 2, de 3 de mayo de 2006, de Educación (preámbulo)

«El interés histórico por la educación se vio reforzado con la aparición de los sistemas educativos contemporáneos. Esas estructuras dedicadas a la formación de los ciudadanos fueron concebidas como instrumentos fundamentales para la construcción de los Estados nacionales, en una época decisiva para su configuración. A partir de entonces, todos los países han prestado una atención creciente a sus sistemas de educación y formación, con el objetivo de adecuarlos a las circunstancias cambiantes y a las expectativas que en ellos se depositaban en cada momento histórico. En consecuencia, su evolución ha sido muy notable, hasta llegar a poseer en la actualidad unas características claramente diferentes de las que tenían en el momento de su constitución.»
LO 5, de 19 de junio de 2002, de las Cualificaciones y de la Formación Profesional (exposición de motivos)

«El derecho a la educación, que el artículo 27 de la Constitución reconoce a todos con el fin de alcanzar el pleno desarrollo de la personalidad humana en el respeto a los principios democráticos de convivencia y a los derechos y libertades fundamentales, tiene en la formación profesional una vertiente de significación individual y social creciente. En esta misma línea y dentro de los principios rectores de la política social y económica, la Constitución, en su artículo 40 , exige de los poderes públicos el fomento de la formación y readaptación profesionales, instrumentos ambos de esencial importancia para hacer realidad el derecho al trabajo, la libre elección de profesión u oficio o la promoción a través del trabajo. En efecto, la cualificación profesional que proporciona esta formación sirve tanto a los fines de la elevación del nivel y calidad de vida de las personas como a los de la cohesión social y económica y del fomento del empleo».

En ambas citas aparece la expresión «formación», pero con connotaciones y significados claramente diferentes. En una de ellas (Ley Orgánica de Educación) por "formación» se entiende un fenómeno diferenciado, aunque íntimamente relacionado con el de educación (son estructuras al servicio de la formación de los ciudadanos) mientras que en la otra por "formación» se entiende un esfuerzo de influencia $o$ inducción al desarrollo de cualificaciones profesionales $\mathrm{y}$, por tanto, netamente educativo. Es decir, en la Ley Orgánica de las Cualificaciones y de la Formación Profesional se identifican, en la práctica, procesos de educación y de formación. 
Es de destacar que, mientras en el preámbulo de la Ley Orgánica de Educación del año 2006, la expresión "educación» aparece unas 100 veces y la expresión «formación» unas 33 veces (es decir, en una relación de tres a una), en la Ley Orgánica de las Cualificaciones y de la Formación Profesional la expresión «educación» aparece sólo en una ocasión, por 32 ocasiones en que se emplea la expresión «formación». Es evidente que, mientras que en el primer texto legal se está aludiendo a dos procesos bien diferenciados («educación» $\mathrm{y}$ «formación»), en el segundo estos dos procesos se subsumen en una única expresión "formación" (invitamos a la lectura de ambos preámbulos de manera completa para que se compruebe esta apreciación).

Un último ejemplo de cuán confuso puede ser el tema de la formación lo tenemos en las tres citas siguientes, orientadas a clarificar qué se entiende por «formación»:

- «Un trabajo sobre sí mismo, libremente imaginado, deseado y perseguido, realizado a través de medios que se ofrecen o que uno mismo se procura» (Ferry, 1991, p. 43).

- «Formación designa principalmente prácticas. Pero estas prácticas son igualmente nombradas con otros términos tales como perfeccionamiento, reciclaje, educación permanente, aprendizaje [...] En todo caso, se trata de formación "para" o "en" algo, generalmente del orden de la formación profesional» (Honore, 1980, p. 20).

- «Un esfuerzo sistemático y planificado para modificar o desarrollar el conocimiento, las técnicas y las actitudes a través de la experiencia de aprendizaje y conseguir la actuación adecuada en una actividad o rango de actividades. Su propósito en el mundo del trabajo es capacitar a un individuo para que pueda realizar convenientemente una tarea o trabajos dados» (Buckley y Caple, 1991).

Estas ambigüedades y fuentes de confusión en torno a la cuestión de la «formación» responden, en nuestra opinión, a diferentes factores y circunstancias. Una primera, y muy evidente, tiene que ver con la traducción de publicaciones foráneas al castellano, convergiendo expresiones y conceptos originalmente distintos y referidos a realidades diferentes en una única expresión castellana: «formación».

Una segunda fuente de confusión es la que se deriva de la coexistencia de tradiciones epistemológicas, corrientes de pensamiento y campos de conocimiento que convergen en el estudio, análisis y reflexión del fenómeno «educativo-formativo» con cierta ignorancia mutua, usurpándose mutuamente expresiones y discursos reflexivos, sin el adecuado alineamiento teórico y conceptual.

Por otra parte, como consecuencia de lo anterior, la tendencia a utilizar, de manera indistinta o intercambiable, las expresiones «educación» $\mathrm{y}$ «formación», para referirse a actividades de carácter educativo con matices diferenciales, y no como fenómenos diferentes en su naturaleza, aunque relacionados.

Para intentar aportar algunas claves que permitan ayudar a resolver estas fuentes de confusión creemos que es necesario abordar dos cuestiones fundamentales y un corolario: 
1) La clarificación terminológica de la expresión española «formación»en relación con los términos equivalentes en otras lenguas.

2) La distinción básica entre lo que puede entenderse por «educación» y por «formación", para lo que se hace necesario (como corolario) esbozar brevemente las diferentes acotaciones teóricas de la idea de «formación», asociadas a las distinciones terminológicas existentes en las dos tradiciones teóricas más relevantes: formación entendida como «Bildung» $y$ formación entendida como «training».

\section{EL PROBLEMA TERMINOLÓGICO ASOCIADO A LA EXPRESIÓN «FORMACIÓN»}

En otras lenguas este problema está mitigado, por cuanto, por ejemplo, lo que en el libro de Buckley y Caple se traduce por «formación», en su versión original se denomina "training». Así, en inglés se diferencia entre "training», «formation» $y$ «education», o en alemán entre "Bildung», «Ausbildung» $\mathrm{y}$ "Erziehung». Nos detenemos sólo en estas dos lenguas por cuanto la primera es determinante por la primacía absoluta del inglés en el mundo académico y la segunda por cuanto representa, quizá, la tradición más importante en cuanto a la reflexión y al análisis de formación (toda la tradición epistemológica que surge en torno a la Bildung alemana).

Claver, Gascó y Llopis $(1996$, p. 263) ya indicaban que «al hablar de formación debemos puntualizar que existen toda una serie de conceptos afines que se entremezclan con éste y que, además, en multitud de ocasiones, originan una confusión en el contenido de todos ellos. Sin pretender entrar en una discusión sobre la idoneidad del vocablo, queremos señalar que, para la mayoría de los autores, existe una identificación entre los términos entrenamiento y formación, correspondiéndose ambos con el vocablo anglosajón training».

Entre nosotros, en español, incorporamos bajo un único término ("formación») tanto lo que los ingleses identifican como "training" y los alemanes "Ausbildung» como lo que que los alemanes identifican como «Bildung» y los ingleses como «formation» (o «Bildung», por cuanto las dificultades de traducción de este término hacen que, en muchas ocasiones, se deje en su versión original alemana). En resumen, dos fenómenos diferentes, claramente separados en lo conceptual, convergen en un único término castellano.

Tal vez sería conveniente introducir la distinción en nuestra lengua, que ya apuntaran Maturana y Nisis (1997), o Del Pozo (1997), entre «formación», por un lado (entendida como «Bildung»), y «capacitación» (entendida como «Ausbildung» $\mathrm{o}$ «training»), por otro.

Consideramos que, una vez que se enlazan ideas y conceptos y tradiciones epistemológicas en principio diferentes, por la mera coincidencia terminológica, el siguiente paso suele ser la identificación de las mismas en un mismo plano conceptual y, finalmente, la extracción de conclusiones, ideas o teorizaciones que, en principio, nada o poco tienen que ver con los planteamientos originales.

En suma, es posible identificar dos posibles interpretaciones de la palabra «formación», una entendida como "Bildung» y otra entendida como «training» o 
«Ausbildung» y que en nuestra lengua, en ocasiones, se ha querido matizar con el uso de la palabra "capacitación».

Volviendo, entonces, a las controversias y confusiones en el marco genuinamente educativo, ¿en qué difieren la "formación», entendida como "Bildung», y la «formación» entendida como "training», "Ausbildung» o "capacitación»?

Para poder entrar a hacer esta clarificación, es preciso dedicar primero unas líneas a recordar la distinción que, desde el campo de las ciencias de la educación, se hace entre "formación» $y$ «educación», por cuanto la distinción terminológica no debe entenderse como simple matización entre diferentes tipos o interpretaciones de un mismo fenómeno («formación»), sino que exige ser interpretada como diferenciación entre dos fenómenos diferentes: educación y formación.

\section{EDUCACIÓN Y FORMACIÓN}

Con frecuencia, como ya denunciaran Runge y Garcés (2011), en el campo de las ciencias de la educación, se acometen empresas como esta en la que pretendemos embarcarnos, de tratar de determinar y precisar determinados conceptos de naturaleza teórica (técnica) educativa, adoptando lo que ellos describen como una cierta "actitud adámica». Esto es, produciendo «saber» como si lo que en ese momento se aporta o escribe fuese algo absolutamente pionero o como si se estuviese descubriendo un campo nuevo, pendiente de explorar. Runge y Garcés (2011) describen, además, la forma frecuente de proceder (que nosotros, aquí, tratamos de evitar) de quienes adoptan esas posiciones «adámicas», en los términos siguientes: «Recurren a los diccionarios de la RAE $\mathrm{u}$ otros de amplia consulta -no especializados, si nos permiten la distinción- para explicar conceptos de la pedagogía que ya tienen una amplia tradición y discusión [...]» (Runge y Garcés, 2011).

Las consecuencias de esta actitud adámica se traducen, para estos autores, en las siguientes (Runge y Garcés, 2011):

1) No nos permite reconocer la tradición de un concepto o su historia -que es la historia de sus problematizaciones- $y$, por tanto, nos impide vincularnos argumentativamente y controversialmente a una tradición de pensamiento - a una comunidad de discusión- y construir una propia.

2) Nuestras producciones de saber no pueden dialogar - o al menos no con facilidadcon otras producciones debido a que los usos conceptuales resultan harto confusos o se encuentran instalados en contextos discursivos disparatados o irreconocibles para colegas de otros países.

3) Se perpetran despropósitos gigantescos que nos dejan en ridículo y nos hacen interlocutores poco creíbles.

Es interesante, por tanto, para evitar incurrir en esa actitud adámica, tratar de enlazar con los conceptos, tradiciones y problematizaciones de las líneas de pensamiento y teorizaciones desarrolladas hasta la fecha, en relación con las diferentes interpretaciones de las expresiones "formación» $\mathrm{y}$ «educación». 
Volviendo a Menze (1980; 269-270), desde la tradición de la reflexión pedagógica alemana: «Educación significa acción desde fuera sobre el hombre en el devenir. Es el conjunto de las medidas necesarias para guiarlo. La formación designa el proceso de desarrollo en el que se manifiesta al exterior algo interior. No es, sin embargo, un proceso de desarrollo a manera de una fuerza que se impone hacia fuera sin quedar contrarrestada por una realidad resistente. La formación tiene lugar en un intercambio con el mundo; no es un proceso orgánico que se lleva a cabo por sí mismo, a la manera del crecimiento de una flor, sino que es inconcebible sin educación».

Esto es, desde esta perspectiva, en síntesis, podemos asumir que educación y formación, siendo fenómenos íntimamente relacionados, son diferentes. El uno alude a un proceso de influencia externa (educación), mientras que el otro alude a un proceso de desarrollo interno (Bildung), propio e íntimo, que se manifiesta externamente (resultado de ese proceso). La idea de formación como Bildung implicaría, en consecuencia, tanto el proceso de crecimiento, aprendizaje, desarrollo y cultivo personal (aculturamiento) como el resultado del mismo.

Sin duda, es en Gadamer (1977) donde podemos encontrar una síntesis más completa y elaborada de la idea de formación (Bildung) desde una perspectiva filosófica. Otros trabajos, como los de Vierhaus (2002) o Vilanou (2001), pueden servir para ofrecer una visión más histórica y social de la génesis y desarrollo de la Bildung alemana. Por otra parte, trabajos como los de Biesta (2002a y b), Litt (1927, 1928) o el de Sianes (2017) nos introducen en la reflexión de las interpretaciones y potencialidades actuales (sin renunciar a una mirada histórica) de la formación, entendida como Bildung.

La re-construcción del complejo núcleo conceptual que implica la Bildung alemana excede, con mucho, las posibilidades de este trabajo (y de estos autores). Para acometer esa empresa sería necesario un recorrido histórico-filosófico, lingüístico y pedagógico que se remontaría hasta la paideia griega. Efectivamente, el ideal educativo-formativo que se relaciona con la Bildung alemana emerge en la sociedad de la antigua Grecia, es posteriormente adoptado por la cultura romana, se integra en la mística medieval y barroca y brota con inusitada potencia en la Ilustración alemana, primero con Kant y luego con Hegel, como camino de ascenso a la humanidad. En esa primera interpretación se estaría aludiendo al «modo específicamente humano de dar forma a las disposiciones y capacidades naturales del hombre» (Gadamer, 1977).

Con Humboldt (2017) se introduce, además, la idea y matización esencial de que no se trata de una forma externamente impuesta, sino que el camino de la formación es un camino propio, libremente elegido, «hasta lograr su propia forma».

Desde esta perspectiva, la formación, entendida como Bildung, se fundamenta en la cualidad humana de la "formabilidad» introducida como elemento discursivo por Herbart (Runge y Garcés, 2011). La «formabilidad» (en alemán "Bildsamkeit»; véase Roth, 1968) es esa condición antropológica que permite al ser humano formarse $y$, por tanto, permite que la educación tenga sentido y, al mismo tiempo, la reclame.

Según Runge y Garcés (2011), la «formabilidad» no debe entenderse como un mecanismo innato, o como un contenido; más bien, como un principio operativo cuya interpretación, desarrollo y alcance siempre estará condicionado por situaciones concretas y contextos específicos. Lo que sí parece claro es que la «formabili- 
dad» no conduce a la formación (Bildung) de manera automática en el ser humano, por el mero hecho de disponer de esta posibilidad, sino que debe ser elicitada, estimulada y fomentada. Ese es el papel que corresponde a la educación.

La consecuencia obvia de este planteamiento, para el ámbito específico de las ciencias de la educación, es que el estudio y profundización de estos dos fenómenos, interrelacionados, exigiría desarrollar, por un lado, una teoría de la formación, entendida como ese proceso de desarrollo y devenir del hombre, de formación sobre sí mismo y nunca acabado; y, por otro lado, una teoría de la educación, entendida como el desarrollo del proceso de influencia que contribuye a orientar y facilitar el proceso de formación (Benner, 1990).

Naturalmente, dentro de esta teoría de la formación cabría distinguir, como ya apuntara Gadamer (1977), una distinción entre formación "general» y formación «especializada» (y, por ello, paralelamente, una distinción entre educación general y educación especializada, que, en cierta medida, coincidiría con lo que anteriormente identificábamos como capacitación o sistemas de formación); o entre formación "teórica» y formación "práctica».

En esta misma línea parece apuntar D. Benner (1990) cuando distingue entre teorías de la formación «formales» y teorías de la formación «materiales» de la manera siguiente: «Las teorías formales de la formación conciben toda clase de contenidos y de ámbitos de actividad como simples materias para la ejercitación y promoción de potencias y facultades humanas; las teorías materiales de la formación, por su lado, ven a los sujetos en proceso de formación únicamente como portadores de aptitudes y cualificaciones socialmente deseables» (Benner, 1990).

En esta distinción empieza a emerger un esquema que parece estar dando acogida a otra forma de entender la formación: la formación como «training».

Al margen de las concepciones derivadas de la tradición filosófica alemana de la Bildung y sus desarrollos posteriores, desde otra perspectiva, Honore (1980, p. 104), al tratar de diferenciar los términos de educación y formación se preguntaba: "iPero por qué hablar de formación para designar lo que podría decirse señalando la importancia cada vez mayor que tienen los aspectos educativos de numerosas actividades, especialmente de la enseñanza [...]? ¿No es la educación la puesta en marcha de todos los medios que permiten al hombre desarrollarse, expresar sus posibilidades, en el medio cultural en el que se encuentra, incluso contribuir al desarrollo y enriquecimiento de ese medio? [...]».

La respuesta a estas cuestiones, del mismo Honore (1980, p. 104), no puede ser más clarificadora: «El término de educación, ha servido durante mucho tiempo para designar la acción de los adultos sobre los niños, para descubrir a la vez y despertar aptitudes y para preparar para su vida de adulto por venir, en las condiciones en las que los adultos viven. Cuando ahora se habla de educación permanente [expresión que Honore emplea como equivalente a la de formación], surge de pronto la imagen de adultos actuando con relación a otros adultos que reemplazan a los nińos».

Desde esta perspectiva, por tanto, lo que habitualmente denominamos «formación» no deja de ser un esfuerzo de naturaleza educativa, de influencia externa, orientado a promover procesos de «formación» interna, especializada, con personas adultas y, generalmente, con connotaciones de desarrollo profesional y adquisición 
de cualificaciones con reconocimientos social-laboral. Es a esta particular forma de acción educativa a la que podemos englobar bajo el término anglosajón de «training» (capacitación, entrenamiento).

Interesa aquí introducir, de manera simétrica a como se hizo al hablar del modelo y corriente asociado a la formación (Bildung) con la idea de «formabilidad» (Bildsamkeit), la idea de «formatividad» (Honoré, 1980, p. 125 y ss.).

$\mathrm{La}$ «formatividad», según Honoré, designa el conjunto de hechos que conciernen a la formación, considerada como función evolutiva del hombre. Para Honoré, la formatividad designa tanto el campo que se deriva de la reflexión sobre la experiencia de actividades que son del orden de la formación como a todas las acciones, todos los hechos, que se relacionan con la función formación.

Especificada de esta manera, la formatividad, en tanto teoría y práctica de la acción, vendría a solaparse con las actividades propias del campo de conocimiento (teoría y acción) de la educación, pero específicamente centradas en las acciones de formación, tal y como las acota Honoré.

Finalmente, añade Honoré, "la formatividad puede también entenderse como el carácter de lo que es formativo, es decir, lo que es una condición favorable, o que ejerce una disposición, un poder para favorecer el proceso de la formación. Representa, pues, la manera en que el entorno material y humano toma las "formas" que, en el medio, sirven de soporte objetivo a la formación” (Honoré, 1980, p. 126).

Para nosotros, en esta última definición se contiene el conjunto de acciones, reflexiones, hechos y principios, de naturaleza educativa, que contribuyen a los procesos de formación del ser humano, en un esfuerzo de integrar en una única teoría de la educación, tanto el ámbito de la educación general como el de las acciones de educación dirigidas al desarrollo de capacidades y cualificaciones más específicas (formación, en sentido restringido, capacitación, o training).

Además de los dos marcos interpretativos de la idea de formación, propios del ámbito de las ciencias de la educación, tratando de aproximarnos a esa peculiar forma de influencia educativa que se desarrolla entre adultos, orientada a promover procesos de capacitación profesional específica, es posible distinguir otros núcleos o aproximación teórico-conceptual: la que se constituye a partir de lo que podríamos denominar el «desarrollo organizacional», economía de la empresa o la administración de recursos humanos, orientado a estudiar la inclusión y desarrollo de estos procesos en el ámbito organizacional-empresarial. Desde esta perspectiva, la formación es siempre planificación de la formación.

Citando a Martín Alcazar: «La formación en el ámbito pedagógico y formación en el marco de la empresa, ni son ni significan lo mismo. En el marco de las empresas la formación pierde sus notas de individualidad, eticidad y libertad para cobrar una dimensión instrumental. Si la formación es fin para la educación, para la economía de las organizaciones es medio. La llamada "formación en la empresa» no es sino formación "para la empresa” (Martín Alcázar, 1999).

De este último núcleo o aproximación conceptual no nos ocuparemos en este trabajo. Baste recordar su existencia como potencial fuente de confusión añadida.

Volviendo, entonces, a los ejemplos que citábamos en apartados anteriores, para clarificar la confusión que rodea a la expresión «formación», haciendo un análi- 
sis a la vista de los comentado hasta ahora, es fácil deducir que cuando Ferry (1991), Honoré (1980) o Buckley y Caple (1991) tratan de definir la "formación» están haciendo referencia a actividades diferentes: en unos casos de naturaleza genuinamente formativa (Bildung) y en otros de naturaleza educativa (influencia externa).

\section{A MODO DE CONCLUSIÓN Y SÍNTESIS}

A modo de síntesis, podemos por tanto concluir que, dentro del campo teórico de las ciencias de la educación, se hace necesario distinguir, por un lado, el ámbito o campo de estudio de la "formación», que englobaría el conjunto de fenómenos relacionados con el desarrollo personal, con el «hacerse persona» y construirse a sí mismo, de naturaleza interna e íntima; y, por otro lado, el ámbito o campo de estudio de la «educación», que aludiría a las prácticas sociales de influencia "externa», educativas, orientadas a elicitar los procesos de formación (internos), mediante dos tipos de prácticas denominadas, respectivamente, «educación» y «formación». Obsérvese que, en este último caso, las prácticas denominadas como "formación», aun recibiendo esa denominación, son de naturaleza «educativa» (si se quiere, instruccional), pero se prefiere esta expresión por su diferente objeto, destinatarios y contextos de desarrollo (capacitación o training). La figura 3 trata de representar, de manera esquemática, estas ideas y distinciones.

Consecuentemente con este planteamiento bidimensional de los campos temáticos de la educación, por un lado, y de la formación por otro, se deriva la consecuencia lógica de la doble articulación, y necesidad, en el marco de las ciencias de la educación, de una "teoría de la formación», por un lado, y de una "teoría de la educación", por el otro.

Tratando de acotar los respectivos cometidos de estas dos orientaciones teóricas, complementarias y mutuamente recíprocas, Benner $(1990,1998)$ establece que la problemática formativa y su elucidación teórica hacen referencia a la meta, al objetivo y al cometido de la actividad pedagógica en todas sus formas y posibilidades. Mientras que la problemática educativa, por el contrario, tiene que ver con el esclarecimiento de los medios y del camino de la interacción pedagógica, y se mueve en el horizonte de una teoría de influjo pedagógico.

Para concluir, queremos hacer una reflexión acerca de la importancia e interés de este esfuerzo de clarificación que trata de representar este trabajo. Comenzábamos haciendo alusión al auge, incremento y omnipresencia de la formación. Hasta el punto de que podemos anticipar que el s. XxI va a ser el siglo de la formación.

No estamos seguros de que esta aceptación e inclusión de los procesos «formativos» en nuestras vidas, la hipertrofia de la oferta y de los presupuestos que se le destinan, estén siendo debidamente sometidas al escrutinio de la crítica. Todo el mundo parte de la premisa de que la formación es algo intrínsecamente bueno, positivo, en lo que merece la pena invertir. ¿Es así?

Creemos que, tal y como señalara Mollenhauer, se hace necesario un posicionamiento crítico de los teóricos de la formación (de la educación) ante el fenómeno: ¿̨formación para qué?, ¿̨formación para quién?, ¿qué formación? 


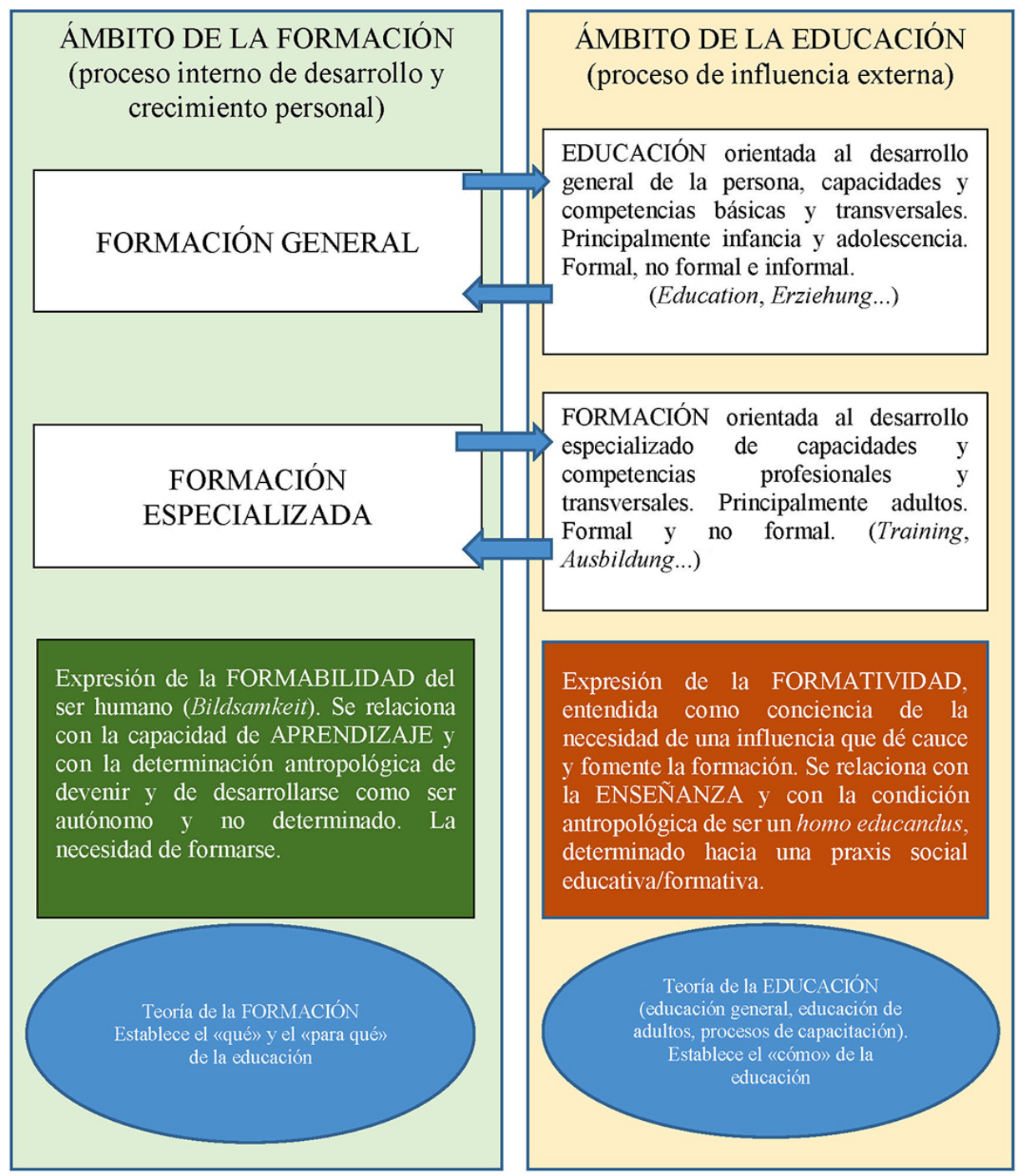

Figura 3. Síntesis esquemática de las diferencias entre los campos conceptuales y teóricos de la educación y la formación, con expresión de sus mutuas interrelaciones.

En este sentido conviene retomar, en este punto, parte del discurso que se ha anticipado en algún momento anterior de este trabajo. La transmutación de la formación, entendida como "tecnología del yo", a una concepción de la formación como «tecnología de producción" $y$, nos tememos, con un solapamiento con lo que Foucault (2008) identificaba como «tecnologías de poder», a la hora de identificar las tecnologías de la razón práctica. 
La formación como tecnología del «yo», entendida como «aquello que permite a los individuos efectuar, por cuenta propia o con ayuda de otros, cierto número de opciones sobre su cuerpo y su alma, pensamiento, conducta o cualquier forma de ser, obteniendo así una transformación de sí mismos con el fin de alcanzar cierto estado de felicidad, pureza, sabiduría o identidad» (Foucault, 2008) (formación-Bildung) sólo puede ser aplaudida, querida y promovida. Ese es el sentido único y legítimo de cualquier acción educativa (formativa). Y con ese altísimo valor y respeto, la expresión «formación» se nos presenta en el ideario colectivo como algo intrínsecamente «bueno», defendible y deseable.

Sin embargo, en nuestra opinión, se corre el peligro de que ese valor colectivo que se le concede a la expresión «formación» sirva de amparo a otras prácticas sociales, también identificadas como "formativas», pero centradas no ya en el desarrollo y crecimiento personal de los individuos que se forman, sino que se ponen al servicio de la producción y dominación. Lo que Foucault (2008) identifica como tecnologías de producción y tecnologías de dominación (normalmente, suelen ir asociadas).

Estas prácticas, alienantes, externalizadas del individuo, como señalara Lyotard (1987), y totalmente desinteresadas de su bienestar, desarrollo personal y, en ningún caso, libremente elegidas no pueden permanecer en el amparo de una percepción benévola, basándose en el término con que se designan,

La confusión terminológica y conceptual es, probablemente, la principal herramienta que facilita este amparo. Esperamos que estas líneas sirvan para poner luz sobre el asunto y animar a que, desde las ciencias de la educación, se articule un discurso teórico y reflexivo crítico sobre estas cuestiones. 


\section{REFERENCIAS BIBLIOGRÁFICAS}

Aguilar, M.C., Martínez, A., Fandiño, A. y Fajardo, S.C. (2007). «Una visión retrospectiva y prospectiva del proceso de formación en las organizaciones». Revista Diversitas-Perspectivas en Psicología, 3 (1), pp. 151-174.

Benner, D. (1990). «Las teorías de la formación: introducción histórico-sistemática a partir de la estructura básica de la acción y el pensamiento pedagógicos». Revista de Educación, 292, 7-36.

Benner, D. (1998). La pedagogía como ciencia. Teoría reflexive de la acción y reforma de la praxis. Barcelona: Pomares-Corredor.

Biesta, G. (2002a). «Bildung and Modernity: The future of Bildung in a World of difference». Studies in Philosophy and Education, 21, 343-351.

Biesta G. (2002b). «How General can Bildung Be? Reflections on the Future of a Modern Educational Ideal». Journal of Philosophy of Education, 36-3, 377-390.

Buckley, R. y Caple, J. (1991). La formación. Teoría y práctica. Madrid: Pirámide.

Claver, E., Gascó, J.L. y Llopis, J. (1996). Los recursos humanos en la empresa: un enfoque directivo. Madrid: Ed. Aranzadi.

Comisión Europea (1996). Enseñar y Aprender. Hacia la Sociedad del Conocimiento. Luxemburgo: Oficina de Publicaciones Oficiales de las Comunidades Europeas.

Del Pozo, P. (1997). Formación de formadores. Madrid: Pirámide.

Ferry, P. (1991). El trayecto de la formación: los enseñantes entre la teoría y la práctica. España: Paidós.

Humboldt, W. (2017). Schriften zur Bildung. Stuttgart: Philipp Reclam jun. GMBH.

Honore, B. (1980). Para una teoría de la formación. Madrid: Narcea.

Foucault, M. (2008). Tecnologías del yo y otros textos afines. Barcelona: Paidós Ibérica.

Gadamer, H.G. (1977). Verdad y método (I). Salamanca: Ed. Sígueme.

Liтt, T. (1927). Die Philosophie der Gegenwart und Ihr Einfluss auf das Bildungsideal. Wiesbaden: Springer.

LitT, T. (1928). Wissenchaft, Bildung, Weltanschauung. Wiesbaden: Springer.

Lyotard, J.F. (1987). La Condición postmoderna. Informe sobre el saber. Madrid: Ediciones Cátedra.

Martín Alcázar, M. (1999). «¡Formación en la empresa? Problemática. Perspectivas». Revista Complutense de Educación, 10 (1); 159-179.

Maturana, H. y Nisis, S. (1997). Formación humana y capacitación. Santiago de Chile: Ediciones Dolmen.

Menze, C. (1980). «Formación», en Speck, J. y Wehle, G. Conceptos fundamentales de Pedagogía. Barcelona: Ed. Herder.

Mollenhauer, K. (1990). «¿Es inevitable corregir el concepto de formación general?». Revista de Educación, 291, 129-148.

Rotr, H. (1968). Pädagogische Anthopologie. Bildsamkeit und Bestimmung. Hannover: Hermann Schroedel Verlag.

RUNGE, A.K. y GARCÉs, F. (2011). «Educabilidad, formación y antropología pedagógica: repensar la educabilidad a la luz de la tradición pedagógica alemana». Revista Científica Guillermo de Ockham, 2; 13-25. 
Sianes, A. (2017). “Bildung”: concepto, evolución e influjo en la pedagogía occidental desde una perspectiva histórica y actual». Revista Española de Educación Comparada, 30; 99-111.

Vierhaus, R. (2002). «Formación (Bildung)». Revista Educación y Pedagogía, vol. xiv-33 (separata); 7-68.

Vilanou, C. (2001). «De la Paideia a la Bildung: hacia una pedagogía hermenéutica». Revista Portuguesa de Educaçao, 14-2, 1-27. 